\title{
Embolia paradójica como causa de enfermedad cerebrovascular isquémica en una mujer joven
}

\author{
Paradoxical embolism as a cause of ischemic \\ cerebrovascular disease in a young female
}

Carrasco-Lozano LE. Embolia paradójica como causa de enfermedad cerebrovascular isquémica en una mujer joven. 2021;34(2): 69-7I. https://doi.org/10.36393/spmi.v34i2.599

\section{Luis Enrique Carrasco-Lozano'}

\begin{abstract}
RESUMEN
Se presenta el caso de una paciente mujer de 44 años sin factores de riesgo, con infarto cerebral extenso y trombosis venosa profunda. En la ecocardiografía transesofágica se evidenció la presencia de un foramen oval permeable, lo cual permitió plantear un caso de embolia paradójica como causa del evento cerebrovascular isquémico. Se descartó otras etiologías probables. La paciente fue dada de alta con mejoría clínica recibiendo anticoagulación, terapia física y rehabilitación.
\end{abstract}

Palabras claves: accidente cerebral vascular, infarto cerebral, foramen oval, embolia paradójica.

\section{ABSTRACT}

This is the case of a 44-year-old female patient with no risk factors, extensive stroke and deep vein thrombosis. Transesophageal echocardiography showed the presence of a permeable oval foramen, which allowed a paradoxical embolism to be raised as a cause of ischemic cerebrovascular event. Other probable etiologies were discarded. The patient was discharged with clinical improvement receiving anticoagulation, physical therapy and rehabilitation. Keywords: vascular stroke, stroke, foramen oval, paradoxical embolism

\section{INTRODUCCIÓN}

A nivel mundial, los eventos cerebrovasculares son la tercera causa de años de vida perdidos por discapacidad (AVDP) para el año 2019 y se encuentran dentro de las 10 primeras causas de AVPD en el grupo etario de 25 a 49 años, superando incluso enfermedades como la tuberculosis, diabetes y enfermedad renal crónica. ${ }^{1}$ Aproximadamente, una de cada cuatro personas mayores de 25 años presentará

Médico internista. Departamento de Medicina Interna, Hospital Nacional Arzobispo Loayza, Lima, Perú. un evento cerebrovascular durante su vida, siendo mayoritariamente de tipo isquémico. ${ }^{2}$

Dentro de las causas de infartos cerebrales en pacientes jóvenes (18-50 años) cobra especial importancia el foramen oval permeable que podría estar asociado incluso hasta a la mitad de casos en pacientes menores de 50 años, por lo que es importante su estudio. ${ }^{3}$ Presentamos el caso de una paciente mujer adulta joven con un infarto cerebral asociado a la presencia de foramen oval permeable.

\section{REPORTE DEL CASO}

Mujer de 44 años, no fumadora, sin antecedentes importantes, ingresó al servicio de Emergencia por trastorno de conciencia, convulsiones tónico-clónicas y hemiplejia izquierda de inicio súbito. El estudio de resonancia magnética nuclear cerebral (RMN) contrastada evidenció una significativa lesión en la región frontotemporal derecha con compromiso de la ínsula y los ganglios basales, con restricción a la difusión, compatible con un infarto (Figura 1).

La paciente fue hospitalizada y en la ampliación de la anamnesis se refirió que la paciente, un año antes, había presentado aumento de volumen del miembro inferior izquierdo, asociado a dolor y limitación de la deambulación. Durante el examen físico, al momento de su ingreso, 


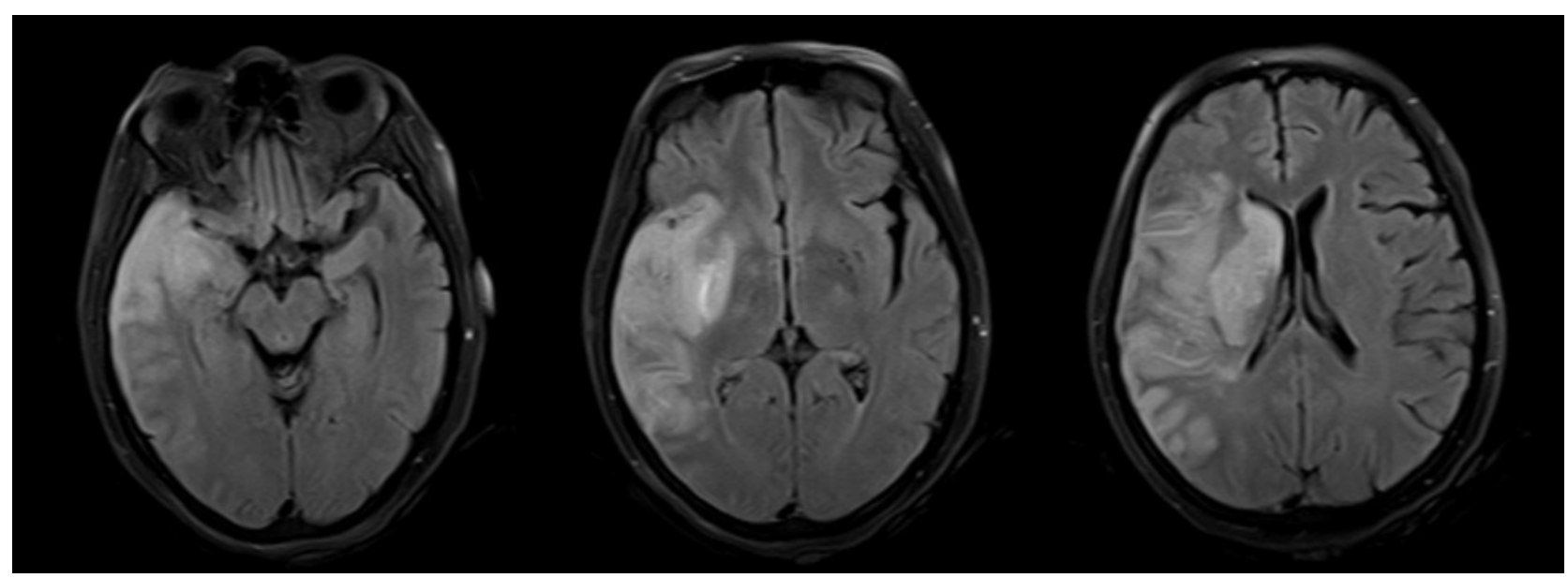

Figura I. Secuencia de difusión en RMN cerebral evidenciando el ictus isquémico. frontotemporal derecho.

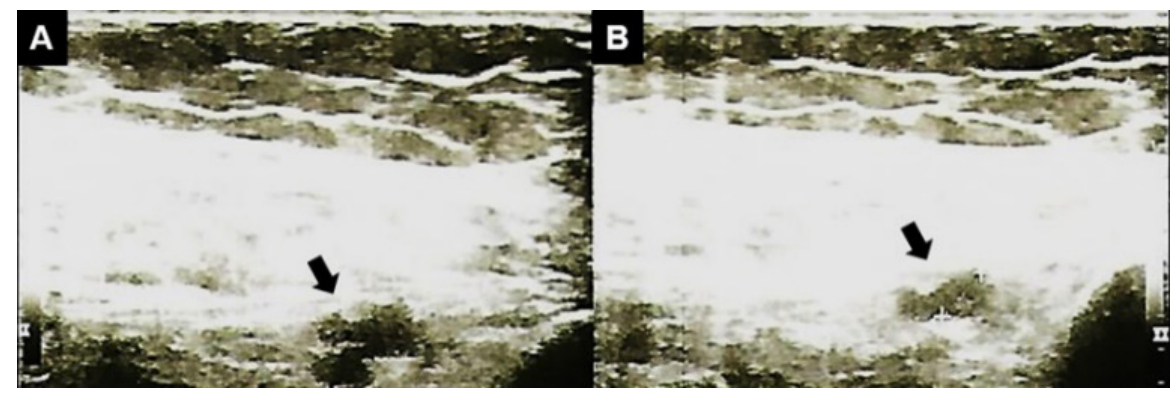

Imagen 2. A. Vena tibial posterior izquierda en ecografía. B. Colapso parcial de vena tibial posterior izquierda en la eco presión con evidencia de trombosis oclusiva.

además de la hemiplejia izquierda se observó asimetría en los miembros inferiores, encontrándose el izquierdo con aumento de volumen. La ecografía de vasos de miembros inferiores mostró la presencia de una trombosis oclusiva en la vena tibial posterior izquierda (Figura 2). El estudio complementario para descarte de síndrome antifosfolipídico, enfermedades autoinmunes y neoplasias ocultas fue negativo.

En el ecocardiograma transtorácico, a nivel de aurícula izquierda anterior se evidenció una imagen de bordes heterogéneos, a considerar un trombo organizado (Figura 3 A). En el posterior estudio de ecocardiograma transesofágico, la aurícula izquierda y su orejuela se encontraron libres de trombos y masas pero se constató la presencia de foramen oval con permeabilidad en el doppler y pasaje de burbujas en el test de suero salino al primer latido de la aurícula derecha a la izquierda (Figuras 3 B-D). La paciente recibió anticoagulación durante la hospitalización con heparina de bajo peso molecular y posteriormente con warfarina siendo dada de alta con un tiempo de protrombina/INR en rango terapéutico. Además, recibió terapia física y rehabilitación, obteniéndose una evolución clínica favorable.

\section{DISCUSIÓN}

Los eventos isquémicos cerebrales constituyen una enfermedad prevalente en personas adultas mayores; sin

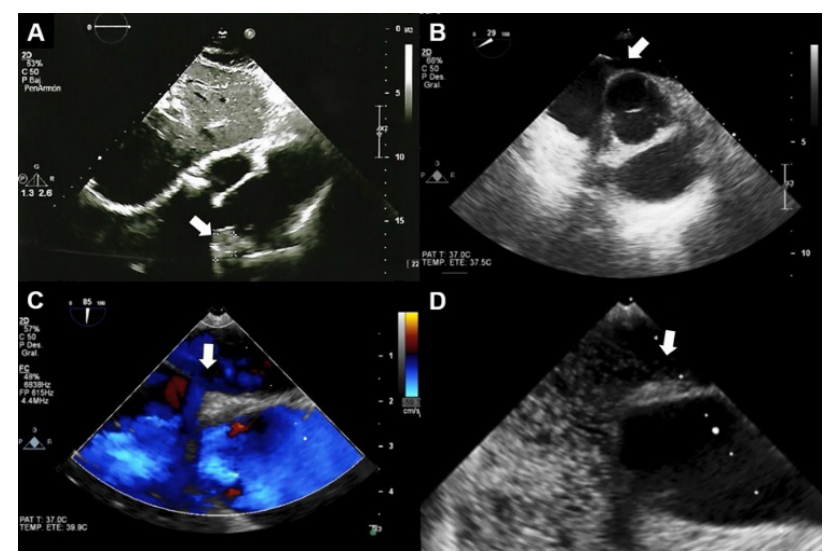

Imagen 3. A. Imagen de bordes heterogéneos en ecocardiograma transtorácico. B. Foramen oval en ecocardiograma transesofágico. C. Estudio doppler con flujo a través del foramen oval. D. Test de suero salino con pasaje de burbujas a través del foramen oval.

embargo, su presentación en pacientes jóvenes puede representar hasta el $20 \%$ del total de casos y se debe tener algunas consideraciones importantes que los diferencian de los pacientes mayores., ${ }^{4,5}$ El grupo etario que define enfermedad cerebrovascular isquémica en paciente joven no está completamente definido pero la literatura coincide en considerar el rango entre 18 y 50 años, por lo cual nuestra paciente debe ser incluida en este grupo., 
Las etiologías a considerar se agrupan en cinco grupos: ateroesclerosis de grandes arterias, cardioembolismo, enfermedad de pequeños vasos, otras etiologías determinadas y de etiología no determinada. ${ }^{5,6}$ El grupo de otras etiologías determinadas comprende causas poco frecuentes de eventos isquémicos cerebrales, y es muy probable que este grupo sea el primero comprendiendo el 26\% de los casos como se representa en The Helsinki Young Stroke Registry que evaluó más de 1000 pacientes, seguido de aquellos pacientes sin causa determinada en un $22,4 \%$ y las causas cardioembólicas en un $19,6 \%{ }^{7}$

Las características imagenológicas de la RMN de la paciente coinciden con una probable etiología cardioembólica debido a la presencia de un compromiso cortical amplio correspondiente a múltiples territorios vasculares. ${ }^{4}$ Dentro de las causas de ictus isquémico con mecanismo cardioembólico en pacientes jóvenes, la principal es el foramen oval permeable hasta en un $44 \%$. $^{7}$ Esta patología es reconocida como uno de los principales factores de riesgo para enfermedad cerebrovascular isquémica en pacientes menores de 50 años e incluso se considera que el $50 \%$ de casos, incluyendo los de etiología no determinada, podría estar asociado a esta condición. ${ }^{3}$

El foramen oval es una comunicación interauricular fisiológica durante la vida fetal que permite el cortocircuito derecha-izquierda necesario durante esta etapa, pudiendo permanecer abierto en los primeros meses de vida. La presencia de un foramen oval permeable, que implica el cierre incompleto de esta comunicación en la vida adulta, puede presentarse en aproximadamente el 25\% de la población. ${ }^{3,8}$ Actualmente es reconocida como una causa de enfermedad cerebrovascular isquémica bajo dos mecanismos principales: la aparición de fibrilación auricular o por embolia paradójica. Este último es más frecuente y constituye el pasaje de un trombo de la circulación venosa a través de la aurícula derecha a la izquierda para llegar a la circulación arterial. Si bien la mayoría de pacientes con foramen oval permeable presentan un cortocircuito izquierda-derecha por la presión mayor a nivel de la aurícula izquierda, eventualmente en circunstancias que eleven la presión de la aurícula derecha se puede generar un cortocircuito derecha-izquierda. ${ }^{3,9}$

En nuestra paciente no se encontró alteración estructural en las aurículas ni se constató fibrilación auricular durante su hospitalización, por lo cual esta posibilidad era bastante alejada, concluyéndose que el mecanismo fisiopatológico de su ictus isquémico fue por embolia paradójica. Además, la presencia en la ecocardiografía de una imagen a nivel de aurícula izquierda que en un posterior control desapareció, indicando que se trataba de un trombo. La literatura describe que para precisar que el foramen oval permeable es la causa del evento isquémico cerebral debe constatarse la presencia de trombo, de lo contrario permanece como una etiología presuntiva mas no determinada. ${ }^{8}$ Ello, sumado a la presencia de trombosis venosa profunda concomitante, considerado un dato clínico importante al plantear un caso de embolia paradójica, que brindó mayor evidencia a que nos encontrábamos frente a un caso de enfermedad cerebrovascular de etiología determinada por un foramen oval permeable..$^{10}$

El tratamiento planteado en estos casos no está del todo claro, extrapolándose datos principalmente de estudios con pacientes con enfermedad cerebrovascular de etiología no determinada o criptogénica que presentaban un foramen oval permeable. ${ }^{9}$ Algunos estudios sugieren que la prevención secundaria con anticoagulantes orales puede ser superior a la antiagregación y que el cierre del defecto anatómico asociado a antiagregación no es superior a la anticoagulación. ${ }^{10}$ En ese sentido, nuestra paciente fue dada de alta con anticoagulación oral con warfarina con adecuado control del tiempo de protrombina/INR, terapia física de rehabilitación neurológica, continuando su control ambulatoriamente constatándose una evolución favorable.

En conclusión, es importante conocer las causas de eventos isquémicos cerebrales en pacientes jóvenes sin factores de riesgo de enfermedad cardiovascular conocidos. Particularmente, cuando el compromiso encefálico es importante debe plantearse la presencia de un foramen oval permeable que puede actuar bajo mecanismo cardioembólico directo por presencia de arritmias auriculares o más frecuentemente mediante embolia paradójica a partir de un trombo en la circulación venosa, como en nuestra paciente.

\section{REFERENCIAS BIBLIOGRÁFICAS}

I. Vos T, Lim SS, Abbafati C, Abbas KM, Abbasi M, Abbasifard M, et al. Global burden of 369 diseases and injuries in 204 countries and territories, 1990-2019: a systematic analysis for the Global Burden of Disease Study 2019. Lancet. 2020 Oct;396(I0258): I 204-22.

2. The GBD 2016 Lifetime Risk of Stroke Collaborators. Global, Regional, and Country-Specific Lifetime Risks of Stroke, 1990 and 2016. N Engl J Med. 20I8 Dec 20;379(25):2429-37.

3. Homma S, Messé SR, Rundek T, Sun Y-P, Franke J, Davidson K, et al. Patent foramen ovale. Nat Rev Dis Primers. 2016 Jan 2I;2(I): I5086.

4. Stack CA, Cole JW. Ischemic stroke in young adults: Curr Opin Cardiol. 2018 Nov;33(6):594-604.

5. Smajlovic D. Strokes in young adults: epidemiology and prevention Vasc Health Risk Manag. 2015 Feb; I57.

6. Ekker MS, Boot EM, Singhal AB, Tan KS, Debette S, Tuladhar AM, et al Epidemiology, aetiology, and management of ischaemic stroke in young adults. Lancet Neurol. 2018 Sep; I7(9):790-80I.

7. Putaala J, Metso AJ, Metso TM, Konkola N, Kraemer Y, Haapaniemi E, et al. Analysis of 1008 consecutive patients aged 15 to 49 with first-ever ischemic stroke:The HelsinkiYoung Stroke Registry. Stroke. 2009 Apr;40(4): I I95-203.

8. Melkumova E, Thaler DE. Cryptogenic stroke and patent foramen ovale risk assessment. Interv Cardiol Clin. 2017 Oct;6(4):487-93.

9. Leppert M, Poisson SN, Carroll JD. Atrial septal defects and cardioembolic strokes. Cardiol Clin. 2016 May;34(2):225-30.

10. Abdelghani M, El-Shedoudy SAO, Nassif M, Bouma BJ, de Winter RJ. Management of patients with patent foramen ovale and cryptogenic stroke:An update. Cardiology. 2019;143(I):62-72.

\section{CORRESPONDENCIA:}

Luis Enrique Carrasco-Lozano,

lecarrascol91@gmail.com

Fecha de recepción: 28-02-2021.

Fecha de aceptación: 24-5-202I.

Financiamiento: autofinanciado.

Conflicto de interés: Nnnguno, según el autor. 